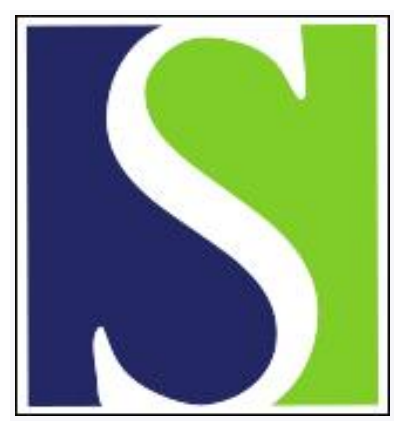

Scand J Work Environ Health 2018;44(1):106-107

https://doi.org/10.5271/sjweh.3697

Published online: 07 Dec 2017, Issue date: 01 Jan 2018

Suicide among agricultural, forestry, and fishery workers

by Shiri $R$

Affiliation: Finnish Institute of Occupational Health, PO Box 18, FI-00032 TYÖTERVEYLAITOS. rahman.shiri@ttl.fi

Refers to the following text of the Journal: 2018;44(1):3-15

The following article refers to this text: 2018;44(1):108-110

Key terms: agriculture; farmer; fishery worker; forestry worker; letter; risk estimate; suicide

This article in PubMed: www.ncbi.nlm.nih.gov/pubmed/29214320

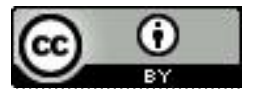




\section{Suicide among agricultural, forestry, and fishery workers}

In their meta-analysis, Klingelschmidt and her associates (1) found that agricultural, forestry, and fishery workers are at $48 \%$ higher risk of suicide than the working-age population. Moreover, they found that the excess risk is even greater among Japanese agricultural workers than workers from other high-income countries. There are several concerns regarding this meta-analysis. It appears that the excess risk has been overestimated for these workers. Furthermore, the excess risk in Japan is not different than other high-income countries.

First, in a systematic review, a literature search is comprehensive. A search of a single database is unlikely to identify most of relevant studies, and these types of reviews are not therefore considered as systematic reviews (2). In this review, a specialized database (PsycINFO) or a European database (EMBASE or Scopus) was not searched.

Second, following the PRISMA guidelines, the critical appraisal of included studies (quality assessment) is a requirement for a systematic review. In a meta-analysis of observational studies, selection bias and confounding should be ruled out.

Third, the reviewers did not correctly extract confidence intervals (CI) for the estimates of several studies such as Hassler 2004, Fleming 1999, and Fragar 2011. Moreover, some studies reported both the least- and maximally adjusted risk estimates. The reviewers, however, extracted age- or the least-adjusted risk estimate. A confounder-adjusted estimate is a more appropriate estimate of the true association. In some studies [eg, Kposowa (3) Agerbo (4)], the excess risk dropped by $52-71 \%$ after adjustment for confounders. As a sensitivity analysis, the reviewers could limit their meta-analysis to a subgroup of studies controlled for confounders.

Fourth, the reviewers did not estimate an overall risk estimate for each study. They included the estimates of 2-6 subgroups for 22 studies in forest and funnel plots. A fixed-effect meta-analysis is a more appropriate model to combine the subgroups of a single study. Moreover, for the assessment of publication bias, it is not appropriate to include several subsamples of a single study in a funnel plot. Using estimates of subgroups can change a large study into several smaller studies.

Fifth, some of the included studies compared agricultural, forestry, and fishery workers with a specific occupational group. The reviewers could calculate a risk estimate using all other occupational groups as a comparison group and exclude those studies that did not provide sufficient data for estimating such a risk estimate. In some studies, the excess risk for agricultural, forestry, and fishery workers disappears after comparing with other occupational groups [eg, adjusted risk ratios (RR) for Kposowa $(3)=1.02,95 \%$ CI $0.41-2.54]$. This is a main reason for observed higher excess risk in Japanese workers. Wada et al (5) compared Japanese agricultural workers with sales workers and Suzuki et al (6) compared Japanese agricultural, forestry, and fishery workers with production process and related workers. Using all other occupational groups as a reference group, age-adjusted RR dropped from 3.53 (95\% CI 2.84-4.38) to 2.61 (95\% CI 2.10-3.25) for Wada et al (5) and from 3.24 (CI 2.95-3.57, both sexes combined) to 1.31 (CI 1.27-1.35 age-adjusted OR after excluding unemployed people) for Suzuki et al (6). The pooled estimate of these two register-based studies was 1.33 (95\% CI 1.29-1.37) using a fixed model and 1.83 (95\% CI 0.93-3.60) using a random model.

Sixth, most of the included studies used register data, which had little information on the background characteristics of the participants. A majority of these studies controlled the estimates for age and sex only. Moreover, in this review, prospective cohort studies did not support the observed association. A meta-analysis of 11 case-control and prospective cohort studies shows no significant excess risk of suicide for agricultural, forestry, and fishery workers (pooled estimate $=1.02$, 95\% CI $0.71-1.47$ for 6 cohort studies and 1.13, 95\% CI $0.92-1.39, \mathrm{I} 2=91 \%$ for 11 case control and cohort studies, combining maximally adjusted risk estimates and comparing agricultural, forestry, and fishery workers with all other occupational groups where possible). The excess risk found in this review (1) can thus largely be due to confounding.

\section{References}

1. Klingelschmidt J, Milner A, Khireddine-Medouni I, Witt K, Alexopoulos EC, Toivanen S, LaMontagne AD, Chastang JF, Niedhammer I. Suicide among agricultural, forestry, and fishery workers: a systematic literature review and meta- 
analysis. Scand J Work Environ Health. 2018;44(1):3-15. https://doi.org/10.5271/sjweh.3682.

2. Puljak L. If there is only one author or only one database was searched, a study should not be called a systematic review. J Clin Epidemiol. 2017;91:4-5. https://doi.org/10.1016/j. jclinepi.2017.08.002.

3. Kposowa AJ. Suicide mortality in the United States: differentials by industrial and occupational groups. Am J Ind Med. 1999;36:645-52. https://doi.org/10.1002/(SICI)10970274(199912)36:6<645::AID-AJIM7>3.0.CO;2-T.

4. Agerbo E, Gunnell D, Bonde JP, Mortensen PB, Nordentoft M. Suicide and occupation: the impact of socio-economic, demographic and psychiatric differences. Psychol Med. 2007;37:1131-40. https://doi.org/10.1017/
S0033291707000487.

5. Wada K, Gilmour S. Inequality in mortality by occupation related to economic crisis from 1980 to 2010 among workingage Japanese males. Sci Rep. 2016;6:22255. https://doi. org/10.1038/srep22255.

6. Suzuki E, Kashima S, Kawachi I, Subramanian SV. Social and geographical inequalities in suicide in Japan from 1975 through 2005: a census-based longitudinal analysis. PLoS One. 2013;8:e63443. https://doi.org/10.1371/journal. pone. 0063443 .

Rahman Shiri

Finnish Institute of Occupational Health

P.O. Box 18, FI-00032

TYÖTERVEYLAITOS

[E-mail: rahman.shiri@ttl.fi] 